Research Article

\title{
State Measurement and Control Technology of Unmanned Vehicle Based on High-Precision Positioning
}

\author{
Zhongyi Zhao and Yongzhi Cui \\ College of Mechanical Engineering and Automation, Liaoning University of Technology, Jinzhou, Liaoning 121001, China \\ Correspondence should be addressed to Yongzhi Cui; jxzzy@lnut.edu.cn
}

Received 20 August 2021; Revised 28 September 2021; Accepted 13 October 2021; Published 31 October 2021

Academic Editor: Sang-Bing Tsai

Copyright (c) 2021 Zhongyi Zhao and Yongzhi Cui. This is an open access article distributed under the Creative Commons Attribution License, which permits unrestricted use, distribution, and reproduction in any medium, provided the original work is properly cited.

\begin{abstract}
For the unmanned vehicle system, the state measurement and control technology is one of the key links to realize the real unmanned vehicle. Based on RTK positioning technology and ultrashort wave spread spectrum communication technology, the key technologies of special vehicles with unmanned automatic operation are studied, and a set of state monitoring and control system of special vehicles with unmanned automatic operation is developed. Through the experimental research, the unmanned vehicle state monitoring and control system can better complete the functions of vehicle location and electronic map display and meet the design requirements. The research results provide technical support for the practical application of special vehicles with unmanned automatic operation.
\end{abstract}

\section{Introduction}

With the rapid development of science and technology in the 21 st century, unmanned intelligent vehicles have become the direction of high-tech development. Its development has great influence on social and economic development, national defense construction, and science and technology, which has become the strategic goal of high-tech research in various countries around the world [1-3]. Driverless technology involves a wide range of research fields, including computer science, automatic control, computer vision, communication technology, and information fusion technology, which promotes the exchange and communication between disciplines, facilitates technology fusion and integration, and also promotes the development of system engineering science [4-6].

Driverless technology can improve the safety of vehicle driving. By installing processors and a variety of sensor devices, unmanned vehicles can realize the perception of road environment, report various dangerous situations to drivers, and give early warning or intelligently and quickly take corresponding safety measures in case of imminent danger $[7,8]$. In industry, agriculture, and fire control, the operation process of high temperature, high pollution, and high risk is not conducive to the use of manual operation, so it is necessary to adopt special vehicles with unmanned automatic operation. In addition, special vehicles with unmanned automatic operation are also used for detection and information collection in harsh and dangerous environments $[9,10]$.

In the research of unmanned vehicle related technology, China is later than the western developed countries. However, with the support of a series of national policies, China's unmanned vehicle and its related technologies have developed rapidly in recent years, but there are deficiencies in key technologies such as environmental perception and vehicle control, which are far behind the level of western developed countries such as the United States. There are still many research problems to be solved before the ultimate goal of thousands of households using unmanned vehicles $[11,12]$. For the unmanned system, the state measurement and control technology is one of the key links to realize the unmanned driving, so it is particularly important to study various key technologies of the unmanned driving state measurement and control system [13]. The state measurement and control technology of special vehicles with unmanned automatic operation is mainly studied, and the experimental verification is carried out. 


\section{System Composition}

The system is mainly divided into two parts: the special vehicle with unmanned automatic operation and the background of the base station. Each background of the base station can connect multiple unmanned vehicles (the specific number is determined by the use environment, observation, and other factors). The special vehicle with unmanned automatic operation includes high-precision positioning, attitude monitoring, air acquisition, image acquisition, data transmission, and other modules. The background base station includes central station system, upper computer, and users. The user connects the central station system through the upper computer to carry out realtime data communication with multiple vehicles.

The central station system is mainly composed of the power management system, the differential source device, and the data processing device. On the one hand, the central station system connects the unmanned vehicle through the data antenna to receive the reported information of the unmanned vehicle and send down the differential information for RTK high-precision positioning; on the other hand, the system connects with the upper computer through the network port to report the position information, air quality information, and video information sent back by the unmanned vehicle and the location information of the reference station in real time. The center station differential source device mainly locates the current base station position through the fixed antenna, generates the RTCM differential source file based on the current base station position, and sends it to the unmanned vehicle for RTK high-precision positioning through the wireless data transmission module.

The system structure of the special vehicle with unmanned automatic operation is shown in Figure 1.

The special vehicle with unmanned automatic operation is composed of positioning, temperature sensing, attitude determination, image acquisition, vehicle chassis control device, air acquisition, and other devices, which are the main elements of on-site investigation. Through high-precision RTK positioning, vehicle position information can be obtained in real time. Through the high-performance data transmission device, the real-time transmission of image data, on-site control data, and other data can be acquired [14].

The structure design of the unmanned vehicle takes into account the installation of positioning antenna, data antenna, image acquisition module, and other structures and ensures the miniaturization and stability design of the whole structure on the basis of no mutual influence of each module. At the same time, the vehicle body has data interface and charging interface, which is convenient for users to charge the vehicle and upgrade the vehicle program.

The 3D model of the unmanned vehicle is shown in Figure 2.

2.1. Power Management System. The DC12 V/32Ah storage battery is used for power supply of the special vehicle with unmanned automatic operation. In order to supply power for the data acquisition and processing module of the vehicle, the system adopts a power management system that combines switching power supply and LDO, step-up module, and step-down module so as to provide stable and reliable power supply for the vehicle and ensure the safe, stable, and reliable operation of each module of the vehicle.

The vehicle power management system is shown in Figure 3.

The power management system is mainly to process the DC12 V power supply to obtain the voltage required by the operation of various parts of the vehicle, which is mainly divided into two circuits: step-up circuit and step-down circuit. The boost part is a Boost module with XL6009EI as the core, and its output circuit can reach $4 \mathrm{~A}$, which mainly supplies power for the wireless transmission module. In the step-down part, the DC-DC module with MP1584 as the core is used to reduce the $12 \mathrm{~V}$ voltage to $5 \mathrm{~V}$ to power the image acquisition device, air acquisition device, RTK positioning device, and other modules, as well as the subsequent LDO. On the basis of $5 \mathrm{~V}$, the LDO is used to output $3.3 \mathrm{~V}$ and $1.8 \mathrm{~V}$ to provide power for MCU data processing center, attitude determination, and other devices.

2.2. High-Precision Positioning Module. RTK (real-time kinematic) technology is a kind of technology that provides real-time dynamic coordinate position by carrier phase difference. Based on the real-time processing of carrier phase observations of two stations, RTK can provide position coordinates for user stations and has high positioning accuracy, which can reach $\mathrm{cm}$ level. In the RTK positioning mode, the observation data of the visual satellite and base station coordinates received by the base station are sent to the mobile station in real time through the wireless data communication link. The mobile station not only needs to receive the visual satellite data but also needs to receive the position information sent by the base station, calculate the baseline length in real time, and give the position coordinates [15].

For the BeiDou Navigation Satellite System (BDS) and GPS satellite system, the positioning solution method is the same, and it is compatible in the aspect of information fusion. BDS/GPS dual-mode carrier phase double difference (DD) data stream passes through the two systems at the same time, and the data processing method is the same as that of the GPS single system.

The high-precision positioning module is shown in Figure 4 .

The high-precision positioning module mainly uses UM442 chip, which supports 432 super channels, has powerful processing ability and embedded JamShield antijamming technology, can track BDS, GPS, GLONASS, Galileo, SBAS, QZSS, and other whole system satellite signals at the same time, and can carry out antijamming processing. Through the enhanced RTK engine calculation, giving full play to the advantages of multiple systems, the centimeter level positioning accuracy and high-precision orientation with high performance and high reliability based on the lowpower and low-cost solution are realized. 


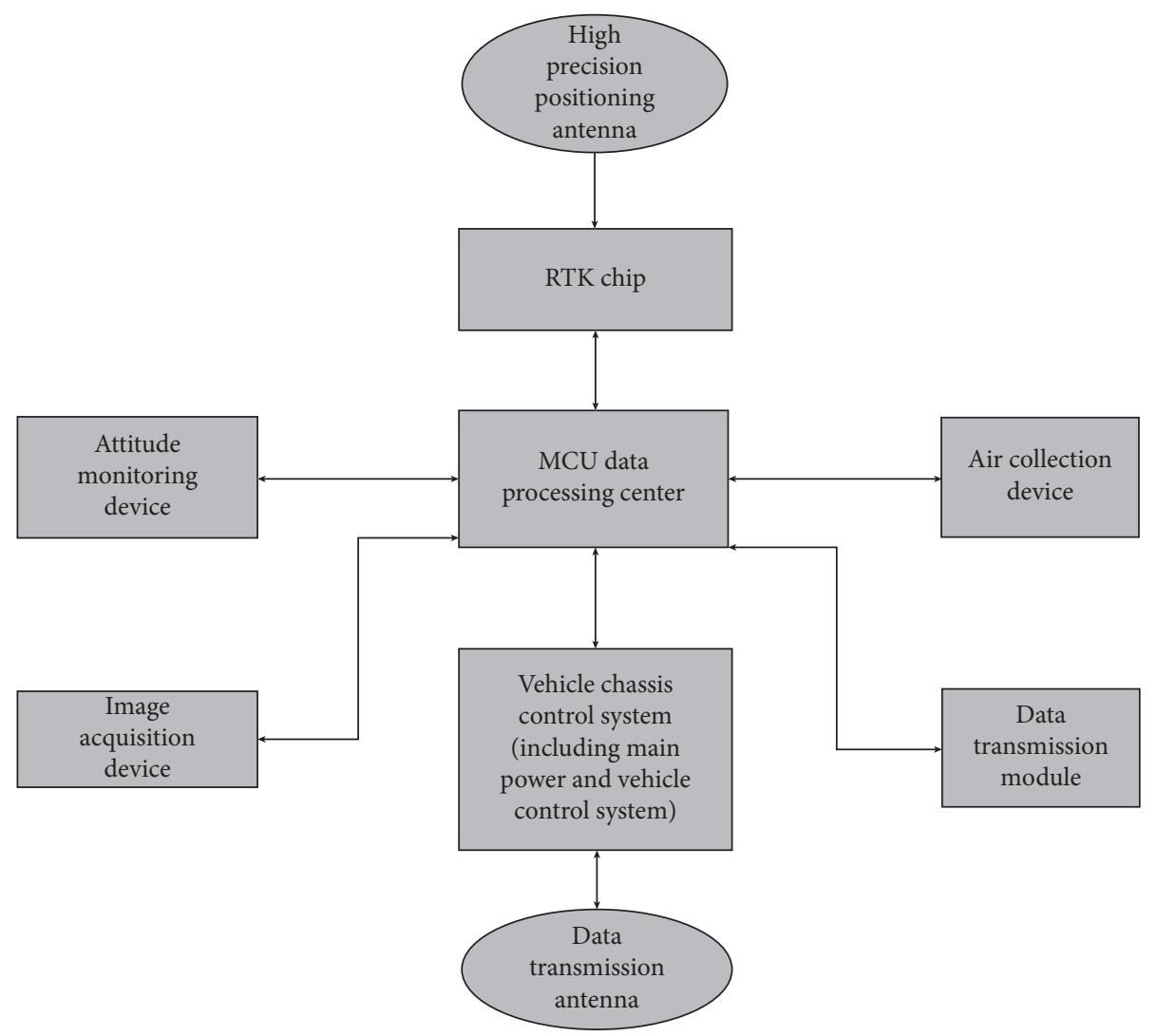

FIgURE 1: Composition block diagram of the unmanned vehicle system.

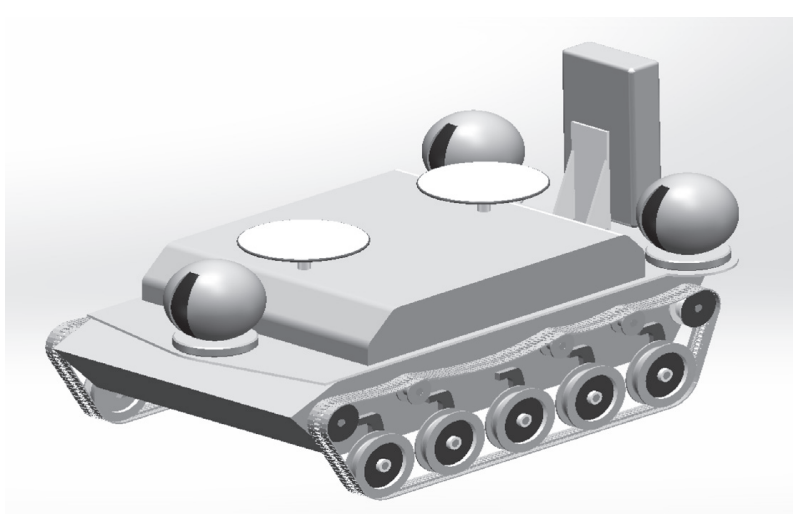

Figure 2: 3D model of the unmanned vehicle.

2.3. Real-Time Attitude Determination Module. The vehicle real-time attitude determination module is mainly used to measure the attitude of the vehicle in the actual movement process so as to play a real-time feedback role on the vehicle's course change, pitch change, and roll change and realize the height prediction combined with the high-precision positioning device.

The attitude determination module integrates JY901 attitude sensor and GNSS dual antenna direction finding module. The attitude sensor integrates attitude solver, adopts advanced digital filtering technology, and uses the mature Kalman filtering algorithm and dynamic algorithm to realize the data fusion of gyroscope, accelerometer, and geomagnetic field sensor so as to realize the real-time motion attitude determination of the module. At the same time, the attitude monitoring device uses the GNSS dual antenna high-precision direction finding module and adopts a more advanced algorithm combining high-precision direction finding and attitude angle to obtain the attitude angle data with higher accuracy and smaller error.

The attitude determination module combined with the attitude sensor and GNSS dual antenna direction finding can collect the attitude data of the vehicle effectively. STM32F103RCT6, as the main processing chip, receives the data uploaded by JY901 for comprehensive processing and completes the network processing of the data together with CH9121.

The attitude sensor is mainly used to measure the threedimensional attitude of the vehicle itself so as to realize the positioning of the environmental position and direction of the vehicle and ensure the position, orientation, and safety of the special operation vehicle.

The vehicle real-time attitude determination module is shown in Figure 5.

2.4. Ambient Air Acquisition Module. The ambient air acquisition module uses S6 + air sensor and barometer to complete the gas analysis of the surrounding environment. S6 + sensor can be used to judge the type and concentration of formaldehyde, $\mathrm{CO}_{2}$, benzene series of toxic vapor, and other toxic gases and can also measure PM2.5, PM10, air 


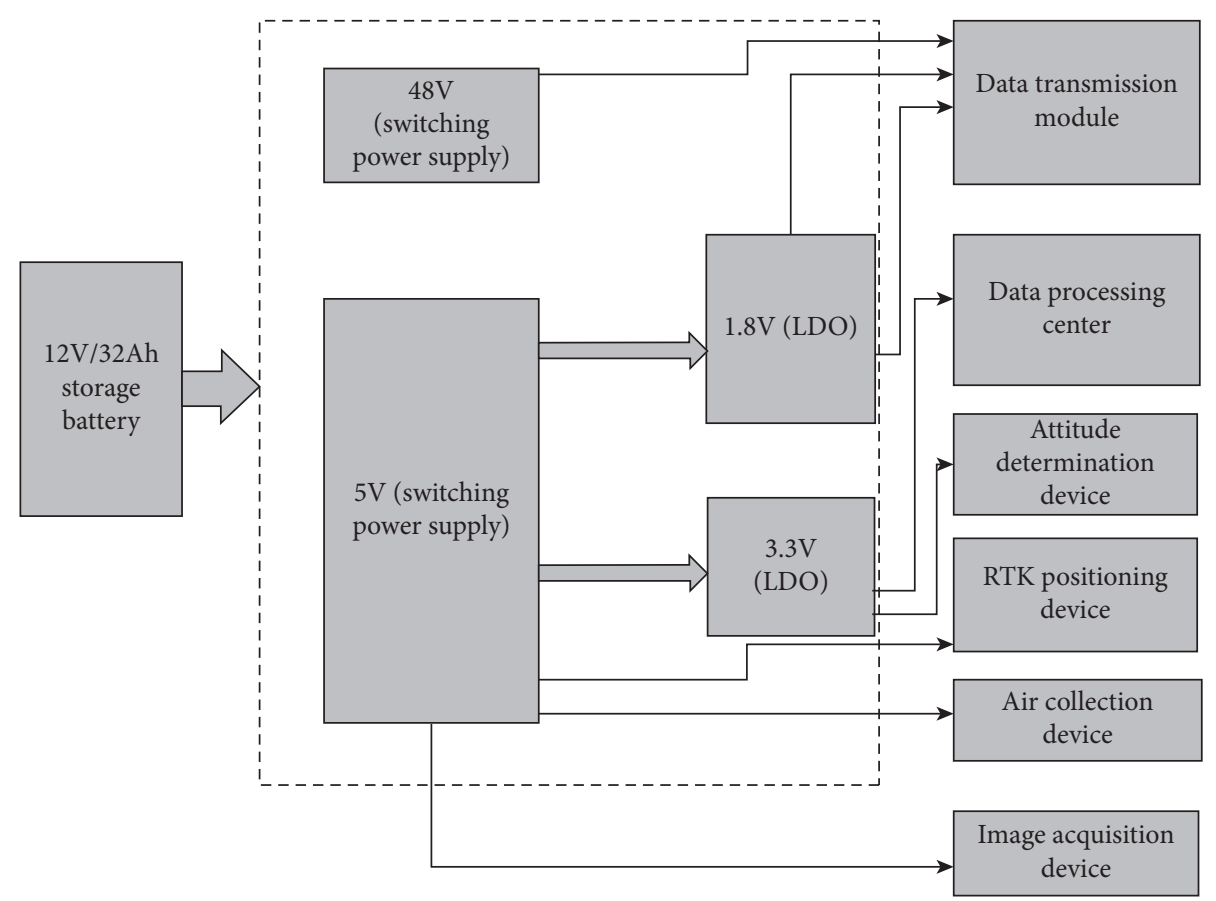

Figure 3: Vehicle power management system.

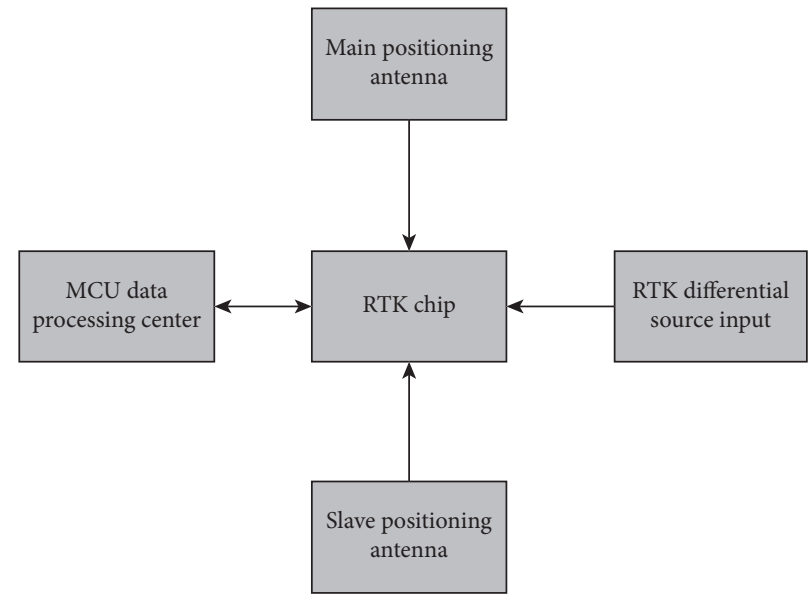

FIGURE 4: Schematic diagram of the high-precision positioning module.

humidity, temperature, and other air quality parameters. At the same time, the high-precision barometer integrated with ambient air acquisition device can monitor the atmospheric pressure of vehicle environment in real time, thus providing a powerful equipment support for field exploration.

The ambient air collection module is shown in Figure 6.

The ambient air data are collected by the ambient air acquisition module and uploaded to the main controller STM32F103RCT6 for data processing. Finally, the IP packet of the data is realized through $\mathrm{CH} 9121$ and transmitted to the corresponding host, so as to achieve the reporting of ambient air quality data.

2.5. Image Acquisition Module. Three rotatable speed dome cameras are used for image acquisition, which are, respectively, placed in the middle of the front and the left and right sides of the rear of the vehicle. Each speed dome camera rotates $340^{\circ}$ horizontally, $105^{\circ}$ vertically upward, and $15^{\circ}$ downward, so as to realize full coverage of $360^{\circ}$ visual angle around the vehicle, and can monitor the surrounding environment of the vehicle in real time.

The installation of the image acquisition module is shown in Figure 7.

2.6. Wireless Data Transmission Module. The unmanned vehicle system uses $5.8 \mathrm{GHz}$ wireless transmission module to realize wireless data transmission, which can realize highspeed data transmission of image, data, and sound. The schematic diagram of wireless data transmission is shown in Figure 8. 


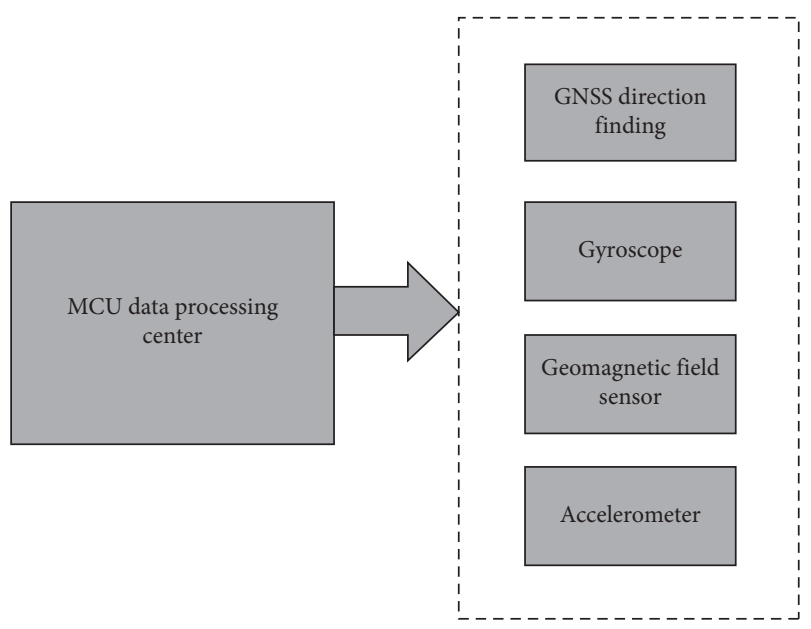

FIGURE 5: Vehicle real-time attitude determination module.

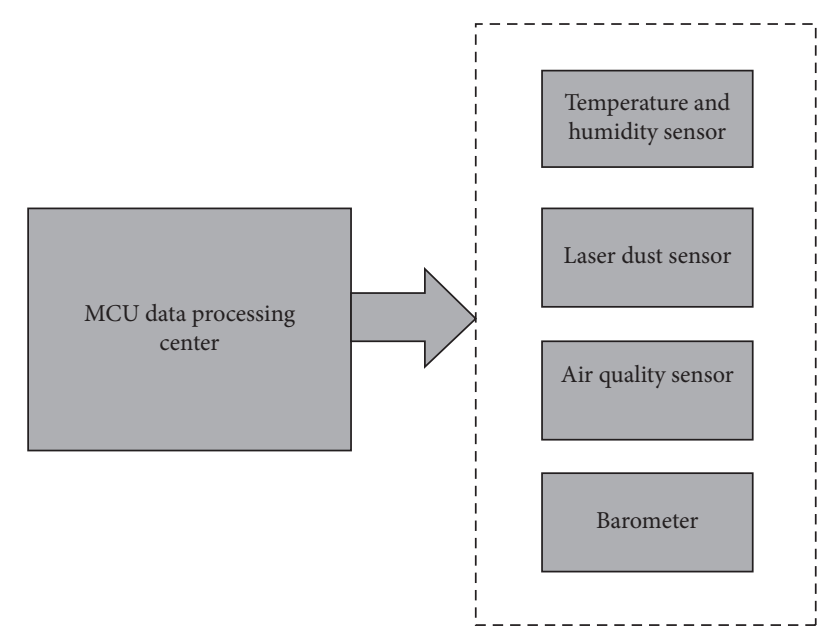

Figure 6: Ambient air acquisition module.

The ultrashort wave spread spectrum communication system is used to transmit the attitude, position, and status information. The spread spectrum communication system enables the system to collect multiple special vehicle operation data and support the application of code division multiple access.

The main characteristics of wireless communication channel are attenuation, slow fading, and all kinds of multipath fast fading. Among them, all kinds of fast fading have the greatest impact on transmission reliability, which are space selective fading, frequency selective fading, and time selective fading. The effective technology to eliminate the influence of channel fading is diversity technology. Wireless diversity technology is a major antifading technology, which can greatly improve the transmission reliability in multipath fading channels.

2.7. Unmanned Vehicle Control System. The unmanned vehicle adopts stainless steel crawler tires. Crawler tires have larger support area, lower ground pressure, and better tractive and appendiculate performance. The turning radius

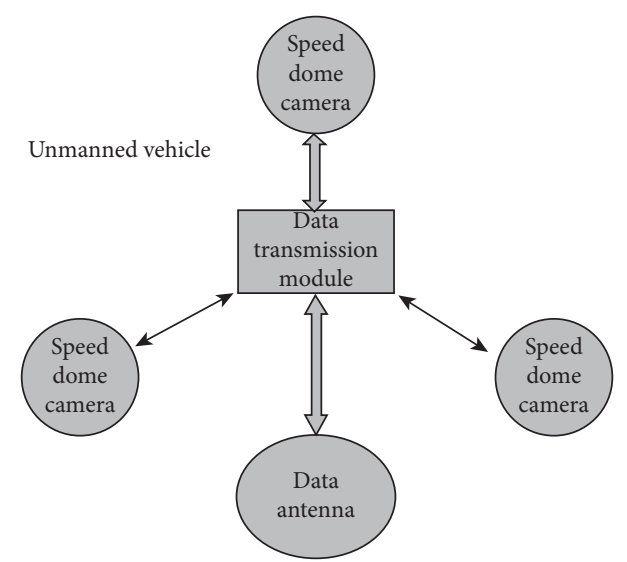

FIGURE 7: Installation diagram of image acquisition module.

of the crawler tires is small, and it is not easy to skid. The crawler tires can overcome the roughness of the ground and has the advantages of good self reset and the ability to climb over obstacles, which can be used in forest, construction site, sandy land, and other fields. At present, they have been widely used in mineral collection, engineering surveying and mapping, framing, disaster relief, and other unstructured environment. Four DC deceleration motors are used to provide power. The $2.4 \mathrm{G}$ encrypted remote controller can ensure the user to operate the vehicle remotely. The schematic diagram of the control system is shown in Figure 9.

DC geared motor, namely, micro geared motor, is composed of static part and rotating part. The static part is called the stator, which consists of a shell and a magnetic pole. Magnetic poles are used to produce magnetic fields. The rotating part is called the rotor, also known as the armature. The armature core is cylindrical, made of laminated silicon steel sheet. The surface is punched with slots, and the armature winding is placed in the slot. The power of the motor running at high speed is reduced by the gear on the input shaft of the gear motor which has fewer teeth meshing with the big gear on the output shaft. The function of the gear reducer is to provide lower speed and greater torque. At the same time, different reduction ratios of the gearbox can provide different speed and torque.

The vehicle and controller adopt $2.4 \mathrm{G}$ encrypted remote controller, and the remote controller has automatic interconnection. When the device is turned on, it will identify the paired device actively, and the communication between the sender and the receiver will be restored automatically after losing the connection. Each pair of remote controllers adopts the encryption algorithm at both ends of the sender and receiver to ensure that multiple devices do not interfere with each other when they are together and lock their paired devices. In addition, the encrypted remote controller has the function of identity recognition. Once the code is matched successfully, it is a one-to-one relationship, and there will be no cross control phenomenon.

In the process of using the vehicle, if the user needs to control the operation of the vehicle, he needs to turn on the remote controller first, and then turn on the power switch of the vehicle. 


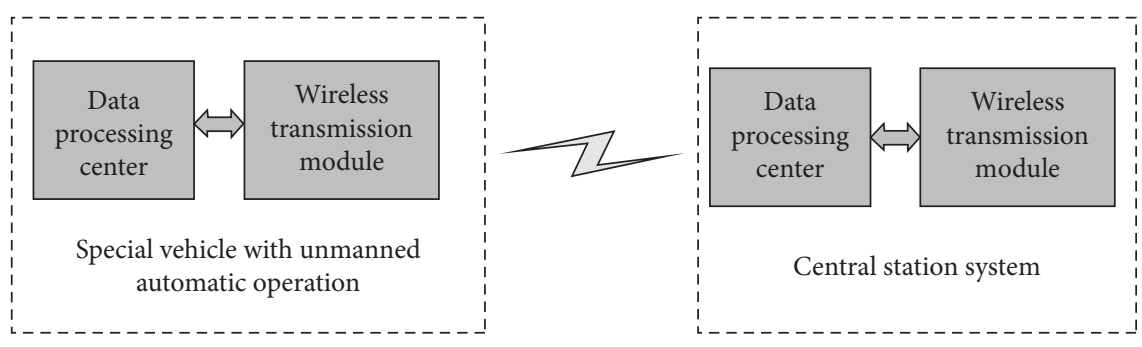

Figure 8: Schematic diagram of data transmission.

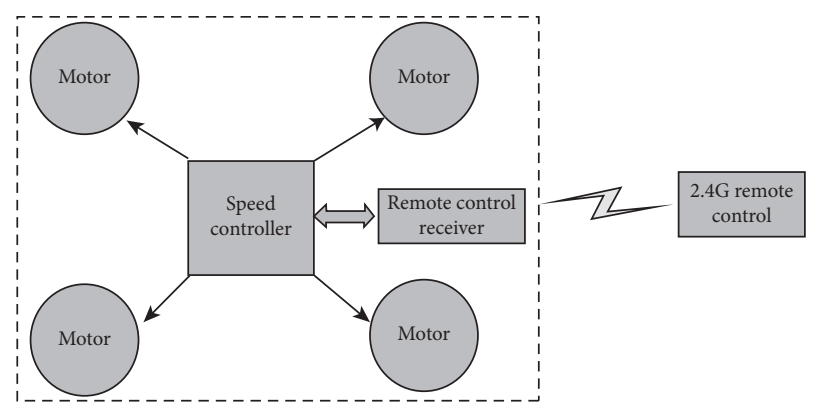

FIgURE 9: Schematic diagram of the control system.

\section{Key Technologies}

3.1. Differential BDS/GPS Technology. Differential GPS (DGPS) firstly uses the differential GPS base station with known precise three-dimensional coordinates to obtain the pseudo range correction or position correction and then sends the correction to the user (GPS navigator) in real time or afterwards to correct the user's measurement data, so as to improve the GPS positioning accuracy. Differential BeiDou composite GPS technology is based on DGPS, comprehensively using BeiDou satellite navigation signal and GPS satellite navigation positioning signal. Through the establishment of BDS/GPS dual-mode multiple base stations (fixed stations), using RTK (real-time kinematic) carrier phase recovery method, the technology can achieve accurate positioning of vehicle position, and its positioning progress can reach centimeter level [16]. However, in complex environments, such as urban forests and mountain canyons, the availability of single system RTK positioning will become worse. With the development of BeiDou Navigation Satellite System in China, GPS (Global Positioning System) in the United States, GLONASS (Global Navigation Satellite System) in Russia, and Galileo in Europe, multisystem integrated positioning can provide more visible satellites than single system positioning, enhance the spatial geometry of satellites, and improve the accuracy, integrity, and reliability of RTK positioning. Therefore, multisystem and multifrequency real-time precision positioning technology will become the development direction of highprecision measurement application of GNSS (Global Navigation Satellite System) in the future.

The mobile station uses the observation data collected to compose the differential observation values to process in real time and gives centimeter level positioning results, which takes less than one second. The mobile station can be in the static state or in the moving state. It can be initialized at a fixed point before entering the dynamic operation, or it can be started directly under the dynamic conditions, and the ambiguity search and solution can be completed in the dynamic environment. After the unknowns of the whole cycle are fixed, the real-time processing of each epoch can be carried out. As long as the tracking of the phase observation values of more than four satellite and the necessary geometric figures are maintained, the mobile station can give centimeter level positioning results at any time [17].

3.2. GIS Display System. Geographic Information System (GIS) is a kind of spatial information and system which can collect, store, manage, analyze, and describe the data related to the whole or part of the earth surface and the spatial geographical distribution. It is a combination of computer science, geography, mapping and remote sensing, environmental science, urban science, space science, information science, and management science. Generally, it has data collection, inspection, and editing functions, data formatting, conversion, and generalization functions, data storage and organization functions, query, retrieval, statistics, and calculation functions, spatial analysis functions, and display functions. The application of GIS has expanded from the early environmental protection and mineral resources management to the water conservancy and electric power, urban planning, land management, transportation, and other areas related to spatial geography [18].

The GIS system used by the system takes high-precision electronic map as the data source, uses the $\mathrm{C} / \mathrm{S}$ architecture system to realize real-time display of the vehicle in the map and real-time playback of the vehicle trajectory, and realizes the function of vehicle's own position judgment and future 


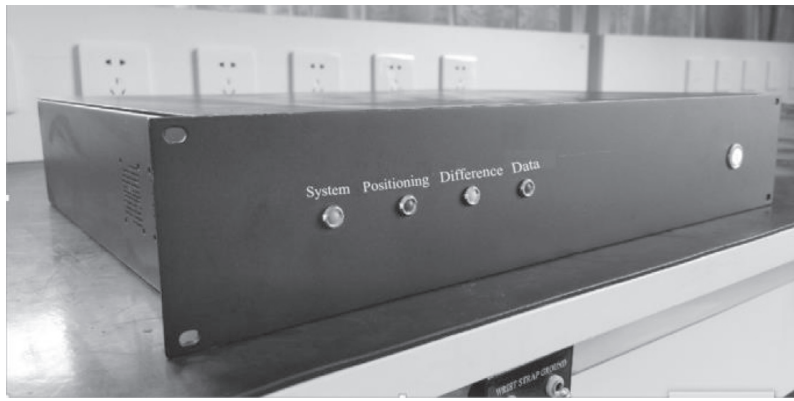

FIgure 10: Physical drawing of the complete machine of the central station.

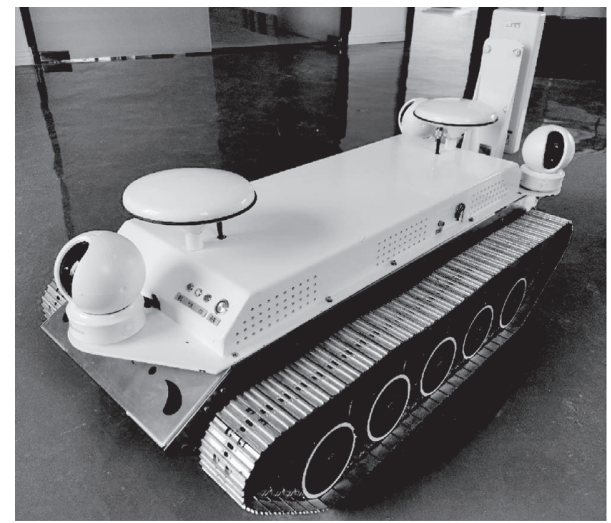

Figure 11: Assembly drawing of the unmanned vehicle.

position prediction by combining the vehicle's OBD bus. At the same time, it can realize the high-precision positioning of the vehicle position by combining the infrared, optical, and radar means of vehicle, which provides the basis for automatic driving.

\section{Experimental Research}

The experimental system is mainly composed of simulation test vehicle, geographic positioning base station, data transmission base station, and command control system. Through the GPS positioning data, attitude data, and environment data collected on the simulation test vehicle, the extended Kalman filter algorithm is used for data processing, the ultrashort wave spread spectrum communication technology is adopted, and data are transmitted to the data center.

4.1. Central Station. The central station is shown in Figure 10 adopting $220 \mathrm{~V}$ AC power supply. The back panel has power input, positioning antenna interface, network interface, POE interface, and other data interfaces. The front panel has system, positioning, difference, data, and other indicator lights, which can directly display the current operation of the central station system. On the one hand, the central station system can generate RTCM differential signal source for the use of unmanned vehicles; on the other hand, it is also the data transfer station between unmanned vehicles and users. It can receive the collected information reported by unmanned vehicles and report it to the upper computer software so that users can intuitively understand the current operation of unmanned vehicles.

4.2. Unmanned Vehicle. The complete assembly drawing of the unmanned vehicle is shown in Figure 11.

4.3. Upper Computer Software. The upper computer software can directly display the information reported by the unmanned vehicle and the central station system in front of the user through graphics and text, which is convenient for the user to intuitively observe the driving state of the vehicle. The software can directly see the air quality index, vehicle running direction, speed, angle, roll angle, and other running states. At the same time, the software integrates electronic map, which can display the current position and historical driving track of the vehicle in real time.

4.4. Application Case. The experimental site is a residential area. The environmental characteristics are as follows: less shelter, complex road environment, and wide field of vision. The initial distance between the base station and the unmanned vehicle is 100 meters, and the maximum linear distance is 400 meters. The motion track, monitoring screen, air quality, vehicle status, and other information are updated in real time. The current location of the base station and the unmanned vehicle and the driving track of the unmanned vehicle are displayed on the map (as shown in Figure 12). 


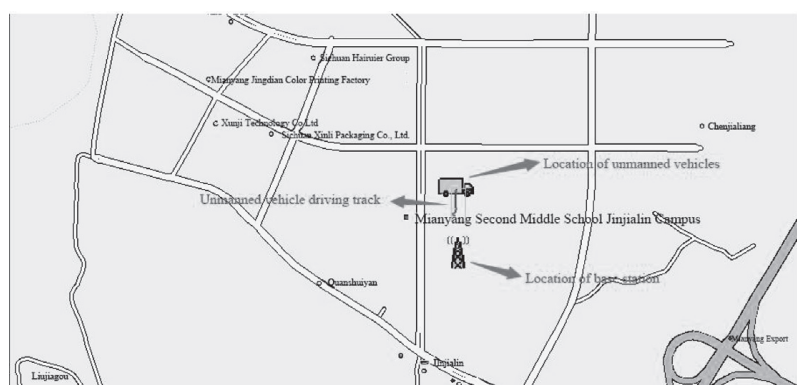

FIGURE 12: Position and track information of the unmanned vehicle.

On the whole, the unmanned vehicle operates normally with good signal reception. The positioning module, air quality acquisition module, and RTK module all work normally with little deviation from the actual situation.

\section{Conclusions}

The state measurement and control technology of the special vehicle with unmanned automatic operation is studied, and the conclusions are as follows.

During the test, the number of BDS and GPS satellites that can be observed is basically more than 8 , which meets the requirements of positioning solution (in order to ensure the accurate three-dimensional position of the unmanned operation vehicle, it is necessary to receive 4 or more effective satellite signals). In the case of short baseline, RTK positioning displays the positioning results from $\mathrm{E}, \mathrm{N}$, and $\mathrm{U}$ directions in the WGS-84 coordinate system. The average error in $\mathrm{E}$ direction is about $2.05 \mathrm{~cm}$; the average error in $\mathrm{N}$ direction is about $3.12 \mathrm{~cm}$; the average error in $\mathrm{U}$ direction is about $2.92 \mathrm{~cm}$, so the positioning accuracy of RTK is in centimeter level, which meets the positioning requirements.

In the static condition, the average error of the course angle of the unmanned vehicle is $0.12^{\circ}$, the average error of the tilt angle is $0.15^{\circ}$, and the average error of the pitch angle is $0.13^{\circ}$. In the dynamic condition, the average error of the course angle is $0.22^{\circ}$, the average error of the tilt angle is $0.24^{\circ}$, and the average error of the pitch angle is $0.23^{\circ}$. The test results are within the error range required by the attitude determination system, which shows that the attitude determination system meets the design requirements.

The use of ultrashort wave makes the communication frequency band have a certain degree of diffraction ability [19]. In the face of complex electromagnetic environment, the spread spectrum communication method is adopted to realize data transmission, which improves the communication anti-interference ability so that the base station can communicate well with the unmanned vehicle [20]. The device can be initialized within 5 seconds, the initialization time is short, and the work efficiency is improved accordingly. The initialization reliability reaches $99.9 \%$, which provides guarantee for the smooth operation of the unmanned vehicle. When the signal loss occurs to the device, the recapture time is less than $1 \mathrm{~s}$, which reduces the cycle slip probability and ensures the positioning accuracy and availability of RTK.

\section{Data Availability}

The unmanned vehicle data and base station data have been uploaded to the attachments.

\section{Conflicts of Interest}

The authors declare that there are no conflicts of interest.

\section{References}

[1] B. W. Zheng and X. M. Xin, Development Demand and Research Significance of Unmanned Combat Vehicle, Shandong Industrial Technology, Zibo, China, 2018.

[2] W. X. Du, C. Yan, W. Xu, and T. Wang, "Implementation and result analysis of RTK algorithm based on GPS/BeiDou network," GNSS World of China, vol. 42, no. 6, pp. 43-47, 2017.

[3] Y. J. Wang, J. Yang, and M. Y. Yu, "Differential positioning algorithm for mobile base station based on BeiDou navigation system," Journal of National University of Defense Technology, vol. 39, no. 57, pp. 46-49, 2017.

[4] X. H. Chen, H. Zu, B. S. Wang, D. E. Zhang, and C. Wu, "Design of high precision positioning service for cooperative vehicle infrastructure system," Laser Journal, vol. 40, no. 11, pp. 109-113, 2019.

[5] J. L. Zhao, X. K. Ling, and Z. S. Li, "Research on high-precision positioning method of BDS/GNSS for android smartphone based on RTK technology," Journal of China University of Technology, vol. 32, no. 2, pp. 110-115, 2020.

[6] S. G. Shen and K. Sun, "Analysis of deformation monitoring applicaiton of Beidou RTK technology in large active bridges," Beijing Surveying and Mapping, vol. 32, no. 12, pp. 1498-1500, 2019.

[7] Z. J. Qi, H. F. Zou, Q. S. Yu, P. Liu, and Y. Zhan, "Research and application of UAV RTK aided positioning technology," Highway, vol. 12, pp. 202-203, 2020.

[8] Z. Jin, G. H. Xia, L. Wang, and X. Zheng, "Research on UAV landing positioning accuracy of UAV based on optimization of power patrol inspection," Equipment Application and Research, vol. 32, no. 602, pp. 26-27, 2019.

[9] Y. C. Lu, D. T. Liang, D. Liang, and P. X. Liu, "Research on inertial navigation/network RTK combined self-localization of outdoor mobile robot," Machine Design and Research, vol. 35, no. 5, pp. 13-17, 2020.

[10] L. F. Guo, X. H. You, Z. X. Su, J. W. Zou, and H. Zou, “Design and research on navigation positioning system of unmanned intelligent vehicle," China Management Informationization, vol. 22, no. 18, pp. 174-176, 2019.

[11] N. X. Bao, Research on RTK Positioning Performance of BeiDou Navigation Satellite System, Civil Aviation University of China, Tianjin, China, 2019.

[12] L. Zhou, Research on Path Planning and Trajectory Tracking Control Method of Tracked Vehicles, Jilin University, Changchun, China, 2020.

[13] Y. Z. Xu, Research on High-Precision Positioning and Navigation System of UAV Based on RTK, Nanjing University of Aeronautics and Astronautics, Nanjing, China, 2018.

[14] R. R. Pan, Research on Agricultural Machinery Precision Positioning System Based on RTK, Zhejiang University, Hangzhou, China, 2017.

[15] L. F. Hao, Research on the Intelligent and Precise Positioning Method Based on BDS, Xidian University, Xian, China, 2019. 
[16] S. J. Zhang, Research on Multi-Sensor Information Fusion Based Localization and Navigation System for Intelligent Vehicle, Harbin Institute of Technology, Harbin, China, 2020.

[17] T. Wang, Research on Localization Method for Autonomous Driving Vehicle, Jilin University, Changchun, China, 2017.

[18] H. Jiang, Research on Automatic Navigation System of Agricultural Machinery Based on RTK Technology, Zhejiang University, Hangzhou, China, 2019.

[19] J. Jackson, B. Davis, and D. Gebre-Egziabher, "An assessment of low-cost RTK GNSS receivers," in Proceedings of the IEEE/ ION PLANSx Meeting, Monterey, CA, USA, April 2018.

[20] T. E. Humphreys, M. J. Murrian, and L. Narula, "Deep urban unaided precise global navigation satellite system vehicle positioning," IEEE Intelligent Transportation Systems Magazine, vol. 12, no. 3, pp. 109-122, 2020. 